\title{
Supplemental Information
}

\section{Experimental Procedures}

Materials. A polyisocyanurate trimer based on isophorone diisociyanate, Tolonate XIDT70B (XIDT, 70\% in butyl acetate), was obtained from Rhodia. XIDT has an isocyanate equivalent weight of 342 g/eq. Polycaprolactone triol (PCL, Tone 0305), having a hydroxyl equivalent weight of 180 g/eq., was obtained from Dow Chemical. Dibutyltin diacetate (DBTDA), 2,4-pentanedione and ethyl 3-ethoxypropionate (EEP) were obtained from Aldrich. Hydroxyl terminated PDMS, $\alpha, \omega-$ bis [3- (2'hydroxyethoxy) propyl] polydimethylsiloxane, FM4411, with hydroxyl equivalent weight 500 g/eq., was obtained from Chisso Corporation. Polyurethane grade methyl n-amyl ketone (MAK) was supplied by Eastman Chemical. Stock solutions of $30 \mathrm{wt} \%$ of PDMS in EEP, 90 wt \% of PCL in MAK, 90 wt \% of Tone 0305 in EEP, 1 wt \% of catalyst dibutyltin diacetate (DBTDA) in MAK were used to prepare formulations. Reagents were used as received without additional purification.

Coating Preparation. For all formulations $0.075 \%$ of DBTDA catalyst and $10 \%$ 2,4pentanedione as pot life extender were added on resin solid basis. $\mathrm{NCO}: \mathrm{OH}$ equivalent ratio was kept constant at 1.1:1.0. For coating preparation all reagents other than the isocyanate were weighed and mixed in a $20 \mathrm{ml}$ vial with magnetic stirring. After thorough mixing, isocyanate was added and mixed well for four hours. Coatings were drawn down over aluminum panels and kept under ambient conditions for 24 hours followed by oven curing at $80^{\circ} \mathrm{C}$ for 45 minutes. Coating film thickness was $50-75$ $\mu \mathrm{m}$. 
Measurements. Atomic force microscopy (AFM) studies were performed on a Dimension $3100^{\circledR}$ microscope with Nanoscope IIIla controller (Veeco, Inc., California). Experiments were carried out by tapping mode in air under ambient condition and also by contact mode under water. Topographical images are presented. Silicon probes with spring constant $0.1-0.4 \mathrm{~N} / \mathrm{m}$ and resonant frequency $17-24 \mathrm{kHz}$ were used. The setpoint ratio for collection of TMAFM data was 0.9 .

Nanoindentation measurements were conducted using the Dimension $3100^{\circledR}$ microscope with using a Berkovich type diamond indenter probe. The spring constant for the probe was $177.3 \mathrm{~N} / \mathrm{m}$. Force-deflection curves from a standard sapphire substrate were used for calibration of deflection voltage and found to average $220.8 \mathrm{~nm} / \mathrm{V}$. A threshold force value of $20 \mu \mathrm{N}$ was used to obtain an array of indentations over the surface of the coating.

For SEM (scanning electron microscopy) experiments, samples were mounted on aluminum mounts and coated with gold using a Technics Hummer II sputter coater. Images were obtained using a JEOL JSM-6300 scanning electron microscope. X-ray information was obtained via a ThermoNoran EDS detector using a VANTAGE Digital Acquisition Engine. 